\title{
MS04-P03 | Efficient Real-Space Refinement For Cryo-Em And Crystallography
}

Urzhumtsev, Alexandre (IGBMC, Illkirch, FRA); Afonine, Pavel V. (LBNL, Berkeley, USA); Poon, Billy K. (LBNL, Berkeley, USA); Read, Randy (Department of Haematology, Cambridge Institute for Medical Research, University of Cambridge, Cambridge, GBR); Sobolev, Oleg V. (LBNL, Berkeley, AUT); Terwilliger, Thomas C. (LANL, Los Alamos, USA); Adams, Paul D. (LBNL, Berkeley, USA)

Recent progress in cryoEM made it possible to refine atomic models against cryoEM maps, $\rho_{\text {exp }}$, at resolutions 2.5-3Å. Usually such real-space refinement calculates a map $\rho_{\text {model }}$ from an atomic model and matches it with $\rho_{\text {exp }}$, e.g. by calculating their correlation value CCmaps. An accurate but computationally expensive way to calculate $\rho_{\text {model }}$ is by obtaining the model Fourier coefficients, truncating them up to the resolution of $\rho_{\exp }$ and calculating the respective Fourier series. Alternatively, $\rho_{\text {model }}$ can be calculated as a sum of contributions of individual atoms where each contribution reflects the resolution of $\rho_{\text {exp }}$. In both cases, the calculation time of the refinement target grows at least as a product of the number of atoms by a cube of the value inverse to the grid step of the map.

We have developed a new refinement procedure phenix.real_space_refine [1] that does not require calculating $\rho_{\text {model }}$ at all thus saving both the computational time and memory. We showed that CCmaps can be approximated by a sum of the $\rho_{\text {exp }}$ values in the positions of atomic centers for any resolution and not only at a very low resolution as suggested earlier [2]. This leads to the refinement algorithm with a very low computational cost independent of the grid size. In turn, this allowed to automate a number of refinement stages, e.g. the weight optimization between the data and restraints terms.

[1] Afonine_et_al.,2018,ActaCryst.,D74,531

[2] Rossmann,2000,ActaCryst.,D56,1341 